\title{
A Novel Approach for Energy Effecient Data Aggregation Using Elephant Behavior as a Swarm Intelligence
}

\author{
M. A. Bharathi, B. P. Vijayakumar, Senior Member, IACSIT, and M. Mallikarjuna
}

\begin{abstract}
A wireless sensor network is a kind of ad hoc network deployed in huge number of nodes in order to sense the physical world. Its application includes both military and civilian services. The sensors are characterized by limited resources and inhibited power hence they experience limited computation, communication and power resources. A major challenge is to provide an improved operational efficiency with limited computational communication and sensing capabilities. In this work we have proposed a clustering technique that groups the sensor node for collaborative processing to enhance the data reliability during aggregation; here we use social behavior of elephants as the biologically inspired swarm intelligence. Simulations are carried out to evaluate the performance of the collaborative behavior that enhances the data aggregation in a resource constrained WSN.
\end{abstract}

Index Terms - Swarm intelligence, cluster, matriarch, data aggregation, social behavior.

\section{INTRODUCTION}

A wireless sensor network is a system of spatially distributed sensor nodes to collect important information about the target environment. A swarm can be viewed as a group of agents cooperating to achieve some purposeful behavior and achieve some goal (Abraham et al., 2006). This collective intelligence seems to emerge from what often large group's .Swarm are computing involves numerous devices that work in a coordinated fashion, communicating either with neighboring devices or the environment to accomplish a defined task. The behavior of a single ant, bee, termite and wasp often is too simple, but their collective and social behavior is of paramount significance. The efforts to mimic such behaviors through computer simulation finally resulted into the fascinating field of SI. SI systems are typically made up of a population of simple agents (an entity capable of performing/executing certain operations) interacting locally with one another and with their environment [1], [2]. Elephants are the largest of land mammals living in very advanced social organizations that require good levels of communication between the groups of individuals. This is because they live in a "fluid fission-fusion" society which simply means that their family units are constantly being

Manuscript received December 12, 2012; revised March 27, 2013. M. A. Bharathi is with Department of Computer Science Engineering, RevaITM Bangalore, India and pursuing PhD in CSE from JNTU Hyderabad India (e-mail: bmalakreddy@yahoo.com).

B. P. Vijayakumar is with Department of Telecommunication Engineering, MSRIT Bangalore, India (e-mail: vijaykbp@yahoo.co.in).

M. Mallikarjun is with Department of Information Science Engineering, RevaITM Bangalore, India (e-mail: mallikarjuna_mathada@yahoo.co.in). divided and reunited whilst, at the same time, they are meeting different individuals on a daily basis.

\section{BACKGROUND AND RELATED WORK}

Swarm intelligence is a field of artificial intelligence that models the intelligent behaviors observed in creature's swarms by using swarm agents. The agents used to model a swarm are often autonomous and self organizing, following a predefined set of rules that govern individual behavior [3]. Ayon Chakraborty et al. [4] have proposed a novel data gathering protocol for enhancing the network lifetime by optimizing energy dissipation in the nodes. To achieve the design objective particle swarm optimization (PSO) with Simulated Annealing (SA) have been applied to form a sub-optimal data gathering chain and devised a method for selecting an efficient leader for communicating to the base station. In the scheme each node only communicates with a close neighbor and takes turns in being the leader depending on its residual energy and location. This helps to rule out the unequal energy dissipation by the individual nodes of the network and results in superior performance as compared to LEACH and PEGASIS [5]. To overcome this problem, a hybrid algorithm based on Genetic Algorithm and Artificial Bee Colony is proposed in this paper. The algorithm resolves the issue through finding the optimal number of clusters, cluster heads and cluster members. Simulation results reveal that this algorithm outperforms LEACH and Genetic Algorithm based clustering scheme. Author [6] discusses about distributed automated sensor deployment algorithms based on swarm intelligence. Author of [7] propose a cluster based private data aggregation, that leverages clustering protocol and algebraic properties of polynomials. Authors of [8]-[10] discusses about robust geographical routing algorithm using aggregator nodes to reduce the energy or power.

\section{PROPOSED WORK}

The work proposed in this paper is an approach for wireless sensor nodes behavior, using biologically inspired swarm intelligence. We try to relate wireless sensor network, using social behavior of elephant's concept in which many players choose to cooperate by forming coalitions. Elephants live in a structured social order. This requires an advanced level of communication and recognition to allow individuals to mediate between the complex relationships that they develop with other individuals. The life of the elephant is very different. As the elephant gets older, it begins to spend more time at the edge of the herd, gradually going off on his 
own for hours or days at a time. Elephants can communicate over long distances by producing and receiving low-frequency sound, a sub-sonic rumbling, which can travel in the air and through the ground much farther than higher frequencies. It is also their ability to learn from interacting with individuals in their intricate social grouping and to recognize important cues for long periods of time which further adds to their intelligence and complex social network. Our work consists of simulating and applying rule abstraction for SI from the below given behaviors of elephant.

1) Female social structure is similar to concentric rings, with the innermost circle comprising a family unit of adult elephants.

2) The most dominant female is called the matriarch. The matriarch is the backbone of the elephant family unit because she provides stability and determines ranging patterns for the rest of the family.

3) As the elephant gets older, it begins to spend more time at the edge of the herd, gradually going off on his own for hours or days at a time.

4) Elephants have a wide range of sounds that they can emit all with different intensities and for different purposes such as securing their defense, attracting mates, co-ordinating movement and generally announcing their needs.

\section{RULES ABSTRACTION}

Abstract Characteristics: We analyze three potential abstract rules for WSN domain: Connectivity, Coverage and Energy. In practical coverage and connectivity depend on energy.

Connectivity: Connectivity represents the overall functionality of the network. A sensor $\mathrm{v}$ is connected to the network $G=(V, E)$, where $\mathrm{V}$ is the set of vertices in the network graph and $\mathrm{E}$ is the set of edges in the network graph. The more connected a network is, the more sensors it has available to monitor the area and supply data. Connectivity is also related to the degree to which the network is partitioned. The figure below discusses about the connectivity in a WSN.

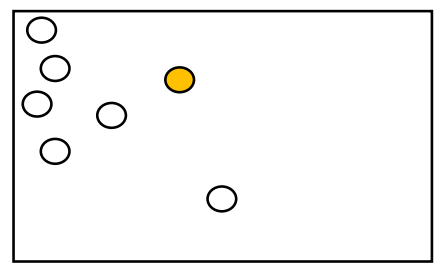

Fig. 1 a. Sensors distributed in an environment. Colored node depicts the sink node.

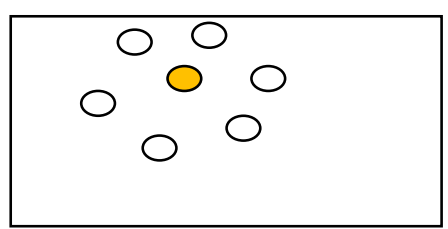

Fig. 1 b. Sensors formed in cluster in an environment in a concentric manner with uniform distribution. Colored node depicts the sink node.

Coverage: Coverage measures how efficiently the network monitors the target area. A region in range of a sensor is only covered if that sensor is itself connected to that network. The algorithm used to compute coverage considers every point in the environment is discussed below.

If there are lot of sensors in the target area

Use a cluster-based protocol

Compute the number of clusters - Social structure

Compute the maximum number of nodes inside each cluster

Elect the cluster leaders - CHs (consider the residual energy and location)

If all cluster leaders are in the range of the Sink Node

Use a one-level hierarchy of cluster-heads (CHs transmit direct to Sink Node)- matriarch.

Else

Use a two or more levels of hierarchy (formation of concentric rings as seen in social structure of elephant) of cluster-heads (function of node density and transmission range).

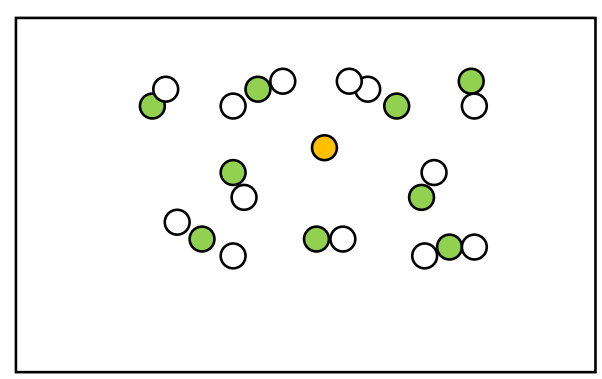

Fig. 2. Cluster of sensors and hierarchy of clusters in an environment. Yellow colored node depicts the sink node and green color nodes the cluster leaders.

Energy: Energy is used to find the connectivity between the nodes and their coverage for transmission and reception of information from the target area.

\section{Rules:}

1) MoveToBorder is a conditional rule that a swarm agent follows when it becomes older. By convention, the agent will move towards the border of the environment grid in an attempt to get isolated from the cluster which simulates the behavior of elephants which move away from the herd as they grow older. The swarm agent will eventually get connected with other agents, provided that the magnitude and sensing/transmission range is reasonable.

2) Slowdown is a simple rule that bounds the maximum speed an agent can attain during the movement of the agent toward the border of the environment.

3) StayatBorders is a simulation specific rule that prevents the sensor agents from moving outside of the target area. When an agent moves within a specific range of the area border, an energy level still exists in them which helps them to be in clusters/groups. If the energy level is too small a node can escape away from the cluster and get towards the border and escape away from the environment, forever isolated from the swarm. For our simulation the boundary value is set at 100 pixels from the sink.

There are three primary ideas that arise in this work that pertain to data aggregation and energy conservation within 
sensor network in the below given cases.

1) A large number of nodes/elephants with high energy levels at the initial phase is that sink's attention will strongly be drawn towards those nodes. Thus the nodes with high energy level convey very relevant information.

2) A small number of node/elephants with moderate energy level after a period of transmissions, is the sinks attention will be drawn, but not as much as in Case 1 .

3) A small number of node/elephants with lowest energy level after a period of transmissions, is the sinks attention will be drawn, less than in above two cases and may even be ignored completely, thinking that the data may be not relevant, because of it low energy level.

\section{Simulation Setup AND Results}

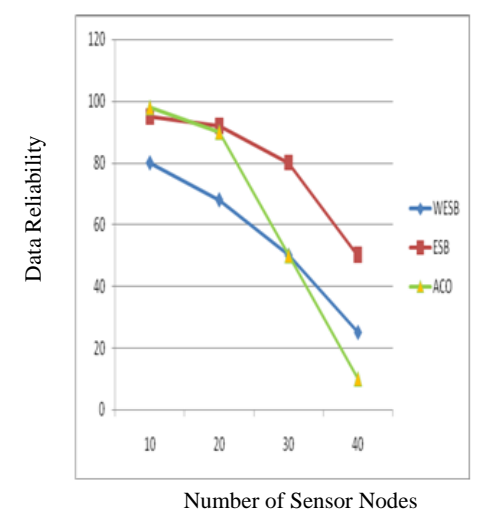

Fig. 3. Graph representing data reliability wrt sensor energy level.

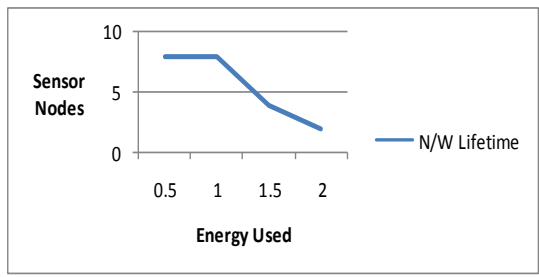

Fig. 4. Graph representing network lifetime.

In the simulation network topology distribution wise layout the slowdown/movetoborder values were choosen from the set $\{0,10,20,30,40,------100\}$ with respect to sink where the sink is placed at the centre. Stay atBorderis where the boundary value is set at 100 pixels from the sink. All combinations of these parameter values were tested with 20 runs for each configuration, each with initial concentric net work layouts to ensure valid results. The graphs in the Fig. 3 shows that the reliability of data used in data aggregation is high when we use elephant social behavior (ESB) when compared with network without using elephants social behavior, by elimination of data sent by the elephants from the border because of their low energy level. Fig. 4 depicts the network life time as the elephants grow older and older they start moving away from the herd and escape from the environment which reduces the lifetime of the network.

\section{CONCLUSION}

Resource constraint environment of WSN requires a energy efficient data aggregation. We have presented a novel swarm abstraction with elephants social behavior model. This model is defined, mapped and applied to wireless sensor network domain, specifically in modeling network characteristics such as connectivity, coverage and energy levels. The above work/investigation has helped to develop new rules and reliability of data for data aggregation with improved energy efficiency and network lifetime.

\section{REFERENCES}

[1] M. K. Jha and T. P. Sharma, "Secure Data Aggregation in Wireless Sensor Networks: A Survey," International Journal of Engineering Science and Technology (IJEST), vol. 5, no. 3, March 2011.

[2] W. Heinzman, A. Chandrakasan, and H. Balakrishnan, "Energy Efficient Communication protocol for Wireless Microsensor Networks," in Proc. the Hawaii International Conference on System Sciences, IEEE, pp. 1-10, January 2000.

[3] D. Miner and M. DesJardins, "Learning Abstract Properties of Swarm Systems," in Proc. 8th International Conference on Autonomous Agents and Multiagent Systems, 2008.

[4] A. Chakraborthy, K. Chakraborthy, S. K. Mitra, and M. K. Naskar, "An Optimized Lifetime Enhancement Scheme for Data Gathering in Wireless Sensor Netwroks," in Proc. ICWCSN, pp. 41-46, 2009

[5] S. Lindsey and C. S. Raghavendra, "PEGASIS: Power Efficient Gathering in Sensor Information Systems," in Proc. IEEE ICC, pp. 1125-1130, June 2001

[6] K. Mittal, A. Veda, and B. K. Meena, "Data Aggregation," Query Processing and Routing in Sensor Networks.

[7] S. Ozdemir and Y. Xiao, "Secure data Aggregation in Wireless Sensor Networks: A comprehensive overview," Computer Networks, vol. 53 , no. 12, 2009.

[8] E. Bonabeau, M. Dorigo, and G. Theraulaz, Swarm Intelligence: From natural to Artificial Systems, Oxford-University Press, 1999.

[9] D. Vass and A. V. Hungary, Distributed Data Aggregation with Geographical Routing in WSN.

[10] V. Mahadevan and F. Chiang, "iACO; A Bio-inspired Power Efficient Routing Scheme for Sensor Neworks," International Journal of Computer Theory and Engineering, vol. 2, no. 6, Dec. 2010.

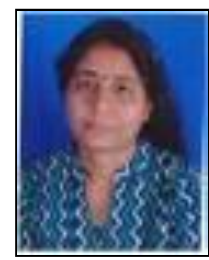

M. A. Bharathi has received her B.E in Computer Science and Engineering from the Gulbarga University Gulbarga, in 1994 and M. Tech from VTU and currently pursuing $\mathrm{PhD}$ from the Department of Computer Science \& Engineering, University of JNTU Hyderabad. Her area of interests are Wireless Sensor Network, Database Management and Adhoc Networks.

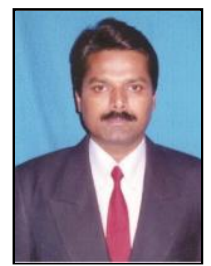

B. P. Vijaya Kumar received $\mathrm{PhD}$ degree in Electrical Communication Engg., Department from Indian Institute of Science (IISc), Bangalore in 2003, M.Tech degree in Computer Science and Technology from Indian Institute of Technology, IITR, with honors in 1992 and Bachelors degree in Electronics and Communications from Mysore University with Distinction in the year 1987. He is currently a Professor and Head, in T elecommunication Engineering Dept. MS Ramaiah Institute of Technology, Bangalore, Karnataka, India, where he is involved in research and teaching UG and PG students, and his major area of research are Computational Intelligence applications in Mobile, Adhoc and Sensor networks.

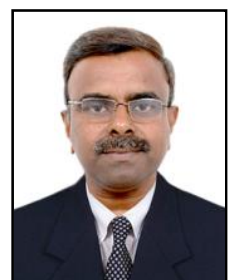

M. Mallikarjuna received his M.Sc (Solid State Physics) and M.Tech (Computer Science) from Jawaharlal Nehru Technological University, Hyderabad. He served as Sr Lecturer in CSE Dept at R $\mathrm{V}$ College of Engineering, Bangalore during the years 1996-2005. He also served as Asst. Professor in the Dept of CSE at BMS Institute of Technology, Bangalore during the years 2005-2008. He is currently working as Sr. Associate Professor in the Dept of ISE at Reva ITM, Bangalore. 